LTH 580

\title{
Three loop anomalous dimension of the second moment of the transversity operator in the $\overline{\mathrm{MS}}$ and $\mathrm{RI}^{\prime}$ schemes
}

\author{
J.A. Gracey, \\ Theoretical Physics Division, \\ Department of Mathematical Sciences, \\ University of Liverpool, \\ P.O. Box 147, \\ Liverpool, \\ L69 3BX, \\ United Kingdom.
}

\begin{abstract}
We compute the anomalous dimension of the second moment of the transversity operator, $\bar{\psi} \sigma^{\mu \nu} D^{\rho} \psi$, at three loops in both the $\overline{\mathrm{MS}}$ and $\mathrm{RI}^{\prime}$ schemes. As a check on the result we also determine the $O\left(1 / N_{f}\right)$ critical exponent of the $n$-th moment of the transversity operator in $d$-dimensions in the large $N_{f}$ expansion and determine leading order information on the $n$ dependence of the anomalous dimension at three and four loops in $\overline{\mathrm{MS}}$. In addition the $\mathrm{RI}^{\prime}$ anomalous dimension of the non-singlet twist-2 operator, $\bar{\psi} \gamma^{\mu} D^{\nu} \psi$, is also determined.
\end{abstract}




\section{Introduction.}

Recently there has been renewed interest in the transversity distribution of the partons in the nucleons. Introduced originally in 1, 2, 3, the transversity measures the difference in probabilities of finding a quark in a transversely polarized nucleon which is polarized parallel to that of the nucleon with that in the antiparallel polarization. Although there has not been as large an experimental activity in this area compared with the conventional distribution functions of deep inelastic scattering, it is possible RHIC may take transversity data in the near future, 4]. (For recent reviews see, for instance, [5, 6].) Therefore, to improve our interpretation of the results it is necessary to extend our theoretical understanding to a similar level as that achieved in deep inelastic scattering. For instance, there has been a large activity in determining the three loop anomalous dimensions of the twist-2 unpolarized and polarized flavour non-singlet and singlet operators as a function of the operator moment, $n$, [7, 8, 9, 10, 11]. These three loop results are necessary for the full two loop renormalization group evolution of the structure functions. Exact analytic results as a function of $n$ are required since the Mellin transform with respect to $n$ determine the parton splitting functions as a function of the momentum fraction, $x$. Such three loop calculations, however, require a huge increase in resources compared with the earlier one and two loop results of [12, 13, 14, 15, 16, 17, 18, 19. For instance, due to the presence of a large number of Feynman diagrams with and without subgraphs, one encounters nested or harmonic sums whose mathematics has had to be studied and developed in, for example, 20]. Moreover, this has then to be encoded in the symbolic manipulation language Form, [21, in order to handle the incredibly large amounts of algebra. Whilst results for non-singlet operators are expected soon it is clear that the same machinery can be applied to determining the anomalous dimensions of the moments of the transversity operator as they differ only in their $\gamma$-algebra structure. For instance, the transversity operator involves the antisymmetric tensor $\sigma^{\mu \nu}=\frac{1}{2}\left[\gamma^{\mu}, \gamma^{\nu}\right]$ instead of one $\gamma$-matrix. Whilst an analytic result for transversity at three loops is some way off, one can turn to the original three loop approach of [7] and determine information on the low moments of the anomalous dimension of the operator. Indeed in [22] the anomalous dimension of the first moment is available at three loops in QCD, building on the two loop result for this tensor current, [23. Moreover, the anomalous dimension of $\bar{\psi} \sigma^{\mu \nu} \psi$ is also available in QED at four loops in $\overline{\mathrm{MS}}$ in the quenched approximation, 24. In this article we will extend 22 by computing the anomalous dimension of $\bar{\psi} \sigma^{\mu \nu} D^{\rho} \psi$ at three loops where $D^{\mu}$ is the usual covariant derivative. Although one motivation for the result lies in the provision of an independent calculation which will be useful to check against the result as a function of $n$ when it is determined, there are several other reasons for concentrating on this one operator. These lie in other theoretical techniques to determine information on the associated matrix element. Using a lattice regularization one can deduce numerical estimates for matrix elements. However, such results are necessarily in a lattice regularization scheme and need to be matched to the standard $\overline{\mathrm{MS}}$ renormalization scheme. Therefore, we will not only determine the anomalous dimension at three loops in $\overline{\mathrm{MS}}$ but also in the $\mathrm{RI}^{\prime}$ scheme which denotes the modified regularization invariant scheme, 25]. This scheme was introduced in 25] and developed for the problem of improving estimates for quark masses in 26, 27]. For applications to similar problems in applying the lattice to construct matrix elements relevant in deep inelastic scattering see, for instance, 28, 29. It is also worth drawing attention to an alternative lattice approach to constructing moments of the parton distribution functions, [30. This uses the Schrödinger functional technique of [31] and is non-perturbative. More recently the $\mathrm{RI}^{\prime}$ scheme has been examined in detail in 32] where QCD has first been renormalized at three loops before reproducing the results of [27] at three loops for the scalar current. Hence the RI' expression for the anomalous dimension of the tensor current was determined to the same order, [32. By contrast to $\overline{\mathrm{MS}}$ the anomalous dimensions of these currents cease to be independent of the covariant gauge parameter. However, only results are 
required in the Landau gauge for the lattice matching. Nevertheless from the point of view of internal consistency in multiloop computations we will perform our calculations in an arbitrary covariant gauge before deducing the requisite conversion functions in the Landau gauge. The disadvantage of an arbitrary gauge calculation is that the computation is algebraically more intense which is further complicated by the fact that the presence of a covariant derivative, itself, introduces another level of integration by parts, also slowing the procedure. Therefore, we have chosen to only focus on the second moment. Choosing the Feynman gauge would speed the computer algebra manipulations but would not be useful for the necessary matching which is gauge dependent. Further, matrix elements for higher moment operators from the lattice are currently much harder to extract.

To be more specific about the results we will report here, we will renormalize both the (flavour non-singlet) operators $\bar{\psi} \gamma^{\mu} D^{\nu} \psi$ and $\bar{\psi} \sigma^{\mu \nu} D^{\rho} \psi$ at three loops in $\overline{\mathrm{MS}}$ and RI'. Although the anomalous dimension of the former operator is known in $\overline{\mathrm{MS}}, 12,13,14,15,16,17,18,19,17$, we will use it as a check on the computer algebra programmes we have written in the symbolic manipulation language Form, 21], before extracting the RI' anomalous dimension. Then the Feynman rule for the operator insertion in a quark two-point function will be replaced with that for the transversity operator. One check on both the $\overline{\mathrm{MS}}$ results will be that the anomalous dimensions of both operators will be independent of the covariant gauge parameter, $\alpha$. The method of calculation is to apply the MINCER algorithm, 33, written in Form, 34, which determines the poles in $\epsilon$ in dimensional regularization, with $d=4-2 \epsilon$, as well as the finite part of massless three loop two-point functions. Each operator will be inserted at zero external momentum to form a two-point function. Moreover, lattice calculations are for the operator inserted at zero momentum so the renormalization scheme conversion functions are required for this configuration. We have used QGRAF, 35, to generate the Feynman diagrams to three loops and the output has been converted to the format used by the FORM version of MincER.

The paper is organised as follows. In section 2 we introduce the method we will use by considering the twist-2 flavour non-singlet operator and reproduce the three loop anomalous dimension for the second moment in $\overline{\mathrm{MS}}$ before deriving the $\mathrm{RI}^{\prime}$ scheme result. This approach is extended in section 3 to the case of the second moment of the transversity operator. The conversion functions which allow one to translate from one renormalization scheme to another for each of the operators we consider are discussed in section 4 and provide a partial check on our results. As an independent check on our three loop result for the transversity we compute the large $N_{f}$ leading order critical exponent for the $n$th moment of the operator using the large $N_{f}$ critical point technique in section 5. Finally, we conclude with discussion in section 6 .

\section{Second moment of non-singlet twist-2 operator.}

As we are considering operator insertions similar to the tensor current which was studied in 22, 32, we briefly recall the RI' renormalization scheme definitions. To determine the correct finite part of the Green's function with the operator inserted we must project out all possible components consistent with Lorentz symmetry. For instance, for the flavour non-singlet operator $\bar{\psi} \gamma^{\mu} D^{\nu} \psi$ we have in momentum space,

$$
\begin{aligned}
G_{\bar{\psi} \gamma^{\mu} D^{\nu} \psi}^{\mu \nu}(p)= & \left\langle\psi(p)\left[\mathcal{S} \bar{\psi} \gamma^{\mu} D^{\nu} \psi\right](0) \bar{\psi}(-p)\right\rangle \\
= & \Sigma_{\bar{\psi} \gamma^{\mu} D^{\nu} \psi}^{(1)}(p)\left(\gamma^{\mu} p^{\nu}+\gamma^{\nu} p^{\mu}-\frac{2}{d} \not p \eta^{\mu \nu}\right) \\
& +\Sigma_{\bar{\psi} \gamma^{\mu} D^{\nu} \psi}^{(2)}(p)\left(p^{\mu} p^{\nu} \not p-\frac{p^{2}}{d} \not p \eta^{\mu \nu}\right)
\end{aligned}
$$


where $p$ is the momentum flowing through the external quark legs and $\mathcal{S}$ denotes both the symmetrization with respect to $\mu$ and $\nu$ and that the operator is traceless*. We could have introduced the null vector, $\Delta_{\mu}$, to achieve this but it is not necessary here as we will in fact reproduce the known anomalous dimension for $\bar{\psi} \gamma^{\mu} D^{\nu} \psi$. To be more specifc

$$
\mathcal{S} \bar{\psi} \gamma^{\mu} D^{\nu} \psi=\bar{\psi} \gamma^{\mu} D^{\nu} \psi+\bar{\psi} \gamma^{\nu} D^{\mu} \psi-\frac{2}{d} \eta^{\mu \nu} \bar{\psi} \gamma^{\sigma} D_{\sigma} \psi
$$

The various components are determined through

$$
\begin{aligned}
\Sigma_{\bar{\psi} \gamma^{\mu} D^{\nu} \psi}^{(1)}(p)=\frac{1}{8(d-1)}[ & \operatorname{tr}\left(\left(\gamma_{\mu} p_{\nu}+\gamma_{\nu} p_{\mu}-\frac{2}{d} \not p \eta_{\mu \nu}\right) G_{\bar{\psi} \gamma^{\mu} D^{\nu} \psi}^{\mu \nu}(p)\right) \\
& \left.-2 \operatorname{tr}\left(\left(p_{\mu} p_{\nu} \not p-\frac{p^{2}}{d} \not p \eta_{\mu \nu}\right) G_{\bar{\psi} \gamma^{\mu} D^{\nu} \psi}^{\mu \nu}(p)\right)\right] \\
\Sigma_{\bar{\psi} \gamma^{\mu} D^{\nu} \psi}^{(2)}(p)=-\frac{1}{4(d-1)}[ & \operatorname{tr}\left(\left(\gamma_{\mu} p_{\nu}+\gamma_{\nu} p_{\mu}-\frac{2}{d} \not p \eta_{\mu \nu}\right) G_{\bar{\psi} \gamma^{\mu} D^{\nu} \psi}^{\mu \nu}(p)\right) \\
& \left.-(d+2) \operatorname{tr}\left(\left(p_{\mu} p_{\nu} \not p-\frac{p^{2}}{d} \not p \eta_{\mu \nu}\right) G_{\bar{\psi} \gamma^{\mu} D^{\nu} \psi}^{\mu \nu}(p)\right)\right] .
\end{aligned}
$$

To renormalize $\left\langle\psi(p)\left[\mathcal{S} \bar{\psi} \gamma^{\mu} D^{\nu} \psi\right](0) \bar{\psi}(-p)\right\rangle$ in our symbolic manipulation approach we follow the method of [36. There the bare Green's function is computed in terms of the bare coupling constant and covariant gauge parameter. Renormalized variables are introduced through the usual renormalization constant definitions such as $g_{\mathrm{o}}=\mu^{\epsilon} Z_{g} g$ where $g$ is the coupling constant appearing in the covariant derivative $D_{\mu}=\partial_{\mu}+i g T^{a} A_{\mu}^{a}, \mu$ is the renormalization scale introduced to ensure the coupling constant remains dimensionless in $d$-dimensions and $T^{a}$ are the generators of the colour group whose structure functions are $f^{a b c}$. The renormalization constant associated with the operator, $Z_{\mathcal{O}}$, is deduced by ensuring that the Green's function is finite after multiplying by $Z_{\mathcal{O}} Z_{\psi}$ where the quark wave function renormalization constant, $Z_{\psi}$, arises from the external quark legs. For the RI' scheme it is the first component which determines $Z_{\mathcal{O}}^{\mathrm{RI}^{\prime}}$ since this renormalization constant is defined by, 25, 32,

$$
\left.\lim _{\epsilon \rightarrow 0}\left[Z_{\psi}^{\mathrm{RI}^{\prime}} Z_{\bar{\psi} \gamma^{\mu} D^{\nu} \psi}^{\mathrm{RI}} \Sigma_{\bar{\psi} \gamma^{\mu} D^{\nu} \psi}^{(1)}(p)\right]\right|_{p^{2}=\mu^{2}}=1
$$

In other words, unlike the $\overline{\mathrm{MS}}$ scheme in addition to the poles in $\epsilon$ the $O(1)$ piece is absorbed into the renormalization constant. Though it is important to note that this renormalization constant will also render the other components finite but not necessarily zero or unity.

Having summarized our method of calculation, we now record our results for the renormalization of $\bar{\psi} \gamma^{\mu} D^{\nu} \psi$. We find

$$
\begin{aligned}
Z_{\bar{\psi} \gamma^{\mu} D^{\nu} \psi}^{\overline{\mathrm{MS}}=} & +\frac{8}{3 \epsilon} C_{F} a \\
+ & {\left[\frac{4}{9}\left(4 T_{F} N_{f}+8 C_{F}-11 C_{A}\right) \frac{1}{\epsilon^{2}}+\frac{4}{27}\left(47 C_{A}-14 C_{F}-16 T_{F} N_{f}\right) \frac{1}{\epsilon}\right] C_{F} a^{2} } \\
+ & {\left[\frac{8}{81}\left(121 C_{A}^{2}-132 C_{A} C_{F}-88 C_{A} T_{F} N_{f}+32 C_{F}^{2}+48 C_{F} T_{F} N_{f}+16 T_{F}^{2} N_{f}^{2}\right) \frac{1}{\epsilon^{3}}\right.} \\
& +\frac{8}{243}\left(718 C_{A} C_{F}-823 C_{A}^{2}+544 C_{A} T_{F} N_{f}-168 C_{F}^{2}\right. \\
& \left.-140 C_{F} T_{F} N_{f}-64 T_{F}^{2} N_{f}^{2}\right) \frac{1}{\epsilon^{2}}
\end{aligned}
$$

${ }^{*}$ The symmetrization and traceless symbol, $\mathcal{S}$, is understood in all our subscripts. 


$$
\begin{aligned}
+\frac{8}{729}( & (648 \zeta(3)+2615) C_{A}^{2}-(1944 \zeta(3)+1066) C_{A} C_{F} \\
& -(1296 \zeta(3)+782) C_{A} T_{F} N_{f}+(1296 \zeta(3)-70) C_{F}^{2} \\
& \left.\left.+(1296 \zeta(3)-853) C_{F} T_{F} N_{f}-112 T_{F}^{2} N_{f}^{2}\right) \frac{1}{\epsilon}\right] C_{F} a^{3}+O\left(a^{4}\right)
\end{aligned}
$$

which is clearly gauge independent and agrees exactly with the result of [7]. Throughout we will present each renormalization constant as an aid to interested readers who wish to perform the check that the double and triple poles in $\epsilon$ can be derived from the simple poles of the previous loop order which follows from the fact that $\overline{\mathrm{MS}}$ is a mass independent renormalization scheme. Further, in (2.5) $\zeta(n)$ is the Riemann zeta function and we have set $a=g^{2} /\left(16 \pi^{2}\right), T^{a} T^{a}=C_{F} I$, $f^{a c d} f^{b c d}=C_{A} \delta^{a b}$ and $\operatorname{Tr}\left(T^{a} T^{b}\right)=T_{F} \delta^{a b}$. Consequently, having checked that our programming reproduces known results it is elementary to change the renormalization scheme and deduce

$$
\begin{aligned}
& Z_{\bar{\psi} \gamma^{\mu} D^{\nu} \psi}^{\mathrm{RI}^{\prime}}=1+\left[\frac{8}{3 \epsilon}+\frac{1}{9}(9 \alpha+31)\right] C_{F} a \\
& +\left[\frac{4}{9}\left(4 T_{F} N_{f}+8 C_{F}-11 C_{A}\right) \frac{1}{\epsilon^{2}}+\frac{4}{27}\left(47 C_{A}+(48+18 \alpha) C_{F}-16 T_{F} N_{f}\right) \frac{1}{\epsilon}\right. \\
& +\frac{1}{324}\left(\left(81 \alpha^{3}+486 \alpha^{2}-324 \zeta(3) \alpha+2439 \alpha-3564 \zeta(3)+12808\right) C_{A}\right. \\
& \left.\left.+\left(162 \alpha^{2}+630 \alpha+2592 \zeta(3)-456\right) C_{F}-(720 \alpha+5336) T_{F} N_{f}\right)\right] a^{2} \\
& +\left[\frac{8}{81}\left(121 C_{A}^{2}-132 C_{A} C_{F}-88 C_{A} T_{F} N_{f}+32 C_{F}^{2}+48 C_{F} T_{F} N_{f}+16 T_{F}^{2} N_{f}^{2}\right) \frac{1}{\epsilon^{3}}\right. \\
& +\frac{4}{243}\left((413-297 \alpha) C_{A} C_{F}-1646 C_{A}^{2}+1088 C_{A} T_{F} N_{f}+(216 \alpha+408) C_{F}^{2}\right. \\
& \left.+(108 \alpha+92) C_{F} T_{F} N_{f}-128 T_{F}^{2} N_{f}^{2}\right) \frac{1}{\epsilon^{2}} \\
& +\frac{2}{729}\left(\left(243 \alpha^{3}+1458 \alpha^{2}-972 \zeta(3) \alpha+9855 \alpha-18468 \zeta(3)+42902\right) C_{A} C_{F}\right. \\
& +(2592 \zeta(3)+10460) C_{A}^{2}-(5184 \zeta(3)+3182) C_{A} T_{F} N_{f} \\
& +\left(486 \alpha^{2}+1134 \alpha-4252+12960 \zeta(3)\right) C_{F}^{2}-448 T_{F}^{2} N_{f}^{2} \\
& \left.+(5184 \zeta(3)-22396-3024 \alpha) C_{F} T_{F} N_{f}\right) \frac{1}{\epsilon} \\
& +\frac{1}{69984}\left(\left(8748 \alpha^{5}+83106 \alpha^{4}-17496 \zeta(3) \alpha^{3}+493776 \alpha^{3}+1659204 \alpha^{2}\right.\right. \\
& -96228 \zeta(3) \alpha^{2}-2523312 \zeta(3) \alpha+174960 \zeta(5) \alpha+7952229 \alpha \\
& -11944044 \zeta(3)+746496 \zeta(4)+524880 \zeta(5)+38226589) C_{A}^{2} \\
& +\left(17496 \alpha^{4}+95256 \alpha^{3}+151632 \zeta(3) \alpha^{2}+416016 \alpha^{2}\right. \\
& +346032 \zeta(3) \alpha+466560 \zeta(5) \alpha+921564 \alpha-4914432 \zeta(3) \\
& -2239488 \zeta(4)+8864640 \zeta(5)+3993332) C_{A} C_{F} \\
& -\left(77760 \alpha^{3}+466560 \alpha^{2}-124416 \zeta(3) \alpha+4091040 \alpha\right. \\
& -369792 \zeta(3)+1492992 \zeta(4)+24752896) C_{A} T_{F} N_{f} \\
& \text { - }\left(155520 \alpha^{2}-622080 \zeta(3) \alpha+1937088 \alpha-3234816 \zeta(3)\right. \\
& -1492992 \zeta(4)+9980032) C_{F} T_{F} N_{f} \\
& +(345600 \alpha+221184 \zeta(3)+3391744) T_{F}^{2} N_{f}^{2} \\
& +\left(17496 \alpha^{3}+289656 \alpha^{2}-373248 \zeta(3) \alpha^{2}-715392 \zeta(3) \alpha\right.
\end{aligned}
$$




$$
\begin{aligned}
& +879336 \alpha+10737792 \zeta(3)+1492992 \zeta(4) \\
& \left.\left.-9331200 \zeta(5)-3848760) C_{F}^{2}\right)\right] C_{F} a^{3}+O\left(a^{4}\right)
\end{aligned}
$$

It is worth commenting on the status of the variables in the renormalization constants in both schemes. We adopt the convention that the scheme is indicated on the renormalization constant itself and therefore the coupling constant and covariant gauge parameter are the variables of the same scheme. However, the three loop renormalization of QCD in the RI' scheme has been given in [32] and the relationship between the parameters has been determined, 32, as

$$
a_{\mathrm{RI}^{\prime}}=a_{\overline{\mathrm{MS}}}+O\left(a_{\overline{\mathrm{MS}}}\right)
$$

and

$$
\begin{aligned}
& \alpha_{\mathrm{RI}^{\prime}}=\left[1+\left((-9 \alpha \overline{\mathrm{MS}}-18 \alpha \overline{\mathrm{MS}}-97) C_{A}+80 T_{F} N_{f}\right) \frac{a_{\overline{\mathrm{MS}}}}{36}\right. \\
& +\left(\left(18 \alpha \frac{4}{\mathrm{MS}}-18 \alpha \frac{3}{\mathrm{MS}}+190 \alpha \frac{2}{\mathrm{MS}}-576 \zeta(3) \alpha \overline{\mathrm{MS}}+463 \alpha \overline{\mathrm{MS}}+864 \zeta(3)-7143\right) C_{A}^{2}\right. \\
& +\left(-320 \alpha \frac{2}{\mathrm{MS}}-320 \alpha \overline{\mathrm{MS}}+2304 \zeta(3)+4248\right) C_{A} T_{F} N_{f} \\
& \left.+(-4608 \zeta(3)+5280) C_{F} T_{F} N_{f}\right) \frac{a_{\overline{\mathrm{MS}}}^{2}}{288} \\
& +\left(\left(-486 \alpha \frac{6}{\mathrm{MS}}+1944 \alpha \frac{5}{\mathrm{MS}}+4212 \zeta(3) \alpha \frac{4}{\mathrm{MS}}-5670 \zeta(5) \alpha \frac{4}{\mathrm{MS}}-18792 \alpha \frac{4}{\mathrm{MS}}\right.\right. \\
& +48276 \zeta(3) \alpha \frac{3}{\mathrm{MS}}-6480 \zeta(5) \alpha \frac{3}{\mathrm{MS}}-75951 \alpha \frac{3}{\mathrm{MS}}-52164 \zeta(3) \alpha \frac{2}{\mathrm{MS}} \\
& +2916 \zeta(4) \alpha \frac{2}{\overline{\mathrm{MS}}}+124740 \zeta(5) \alpha \frac{2}{\overline{\mathrm{MS}}}+92505 \alpha \frac{2}{\mathrm{MS}}-1303668 \zeta(3) \alpha \overline{\mathrm{MS}} \\
& +11664 \zeta(4) \alpha \overline{\mathrm{MS}}+447120 \zeta(5) \alpha \overline{\mathrm{MS}}+354807 \alpha_{\overline{\mathrm{MS}}}+2007504 \zeta(3) \\
& +8748 \zeta(4)+1138050 \zeta(5)-10221367) C_{A}^{3} \\
& +\left(12960 \alpha \frac{4}{\mathrm{MS}}-8640 \alpha \frac{3}{\mathrm{MS}}-129600 \zeta(3) \alpha \frac{2}{\mathrm{MS}}-147288 \alpha \frac{2}{\mathrm{MS}}\right. \\
& +698112 \zeta(3) \alpha \overline{\mathrm{MS}}-312336 \alpha \overline{\mathrm{MS}}+1505088 \zeta(3)-279936 \zeta(4) \\
& -1658880 \zeta(5)+9236488) C_{A}^{2} T_{F} N_{f} \\
& +\left(248832 \zeta(3) \alpha \frac{2}{\overline{\mathrm{MS}}}-285120 \alpha_{\overline{\mathrm{MS}}}^{2}+248832 \zeta(3) \alpha \overline{\mathrm{MS}}-285120 \alpha \overline{\mathrm{MS}}\right. \\
& -5156352 \zeta(3)+373248 \zeta(4)-2488320 \zeta(5)+9293664) C_{A} C_{F} T_{F} N_{f} \\
& +\left(-38400 \alpha \frac{2}{\overline{\mathrm{MS}}}-221184 \zeta(3) \alpha \overline{\mathrm{MS}}+55296 \alpha \overline{\mathrm{MS}}\right. \\
& -884736 \zeta(3)-1343872) C_{A} T_{F}^{2} N_{f}^{2} \\
& +(-3068928 \zeta(3)+4976640 \zeta(5)-988416) C_{F}^{2} T_{F} N_{f} \\
& \left.\left.+(2101248 \zeta(3)-2842368) C_{F} T_{F}^{2} N_{f}^{2}\right) \frac{a_{\overline{\mathrm{MS}}}^{3}}{31104}\right] \alpha \overline{\mathrm{MS}}+O\left(a \frac{4}{\mathrm{MS}}\right)
\end{aligned}
$$

where the scheme of the variable is indicated as a subscript and we note also that the $\mathrm{RI}^{\prime}$ and $\overline{\mathrm{MS}} \beta$-functions are formally equivalent at four loops, 32].

From these renormalization constants we can deduce the anomalous dimensions from

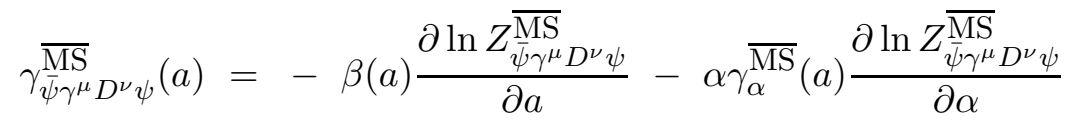

and

$$
\gamma_{\bar{\psi} \gamma^{\mu} D^{\nu} \psi}^{\mathrm{RI}^{\prime}}(a)=-\beta(a) \frac{\partial \ln Z_{\bar{\psi} \gamma^{\mu} D^{\nu} \psi}^{\mathrm{RI}^{\prime}}}{\partial a}-\alpha \gamma_{\alpha}^{\mathrm{RI}^{\prime}}(a) \frac{\partial \ln Z_{\bar{\psi} \gamma^{\mu} D^{\nu} \psi}^{\mathrm{RI}^{\prime}}}{\partial \alpha}
$$


We find, in our conventions,

$$
\begin{aligned}
\gamma_{\bar{\psi} \gamma^{\mu} D^{\nu} \psi}^{\overline{\mathrm{MS}}}(a)=\frac{8}{3} C_{F} a+ & \frac{8 C_{F}}{27}\left[47 C_{A}-14 C_{F}-16 T_{F} N_{f}\right] a^{2} \\
+\frac{8 C_{F}}{243}[ & (648 \zeta(3)+2615) C_{A}^{2}-(1944 \zeta(3)+1066) C_{A} C_{F} \\
& -(1296 \zeta(3)+782) C_{A} T_{F} N_{f}+(1296 \zeta(3)-70) C_{F}^{2} \\
& \left.+(1296 \zeta(3)-853) C_{F} T_{F} N_{f}-112 T_{F}^{2} N_{f}^{2}\right] a^{3}+O\left(a^{4}\right)
\end{aligned}
$$

which agrees with [7] and

$$
\begin{aligned}
\gamma_{\bar{\psi} \gamma^{\mu} D^{\nu} \psi}^{\mathrm{RI}^{\prime}}(a)=\frac{8}{3} C_{F} a+ & \frac{C_{F}}{54}\left[\left(27 \alpha^{2}+81 \alpha+1434\right) C_{A}-224 C_{F}-504 T_{F} N_{f}\right] a^{2} \\
+\frac{C_{F}}{1944}[ & \left(486 \alpha^{4}+4131 \alpha^{3}-972 \zeta(3) \alpha^{2}+19899 \alpha^{2}-10044 \zeta(3) \alpha\right. \\
& +64098 \alpha-115344 \zeta(3)+806800) C_{A}^{2} \\
& -\left(1458 \alpha^{2}+15066 \alpha+10368 \zeta(3)+172856\right) C_{A} C_{F} \\
& -\left(4320 \alpha^{2}-2592 \zeta(3) \alpha+22248 \alpha+25920 \zeta(3)+534400\right) C_{A} T_{F} N_{f} \\
& +(3888 \alpha+41472 \zeta(3)-43328) C_{F} T_{F} N_{f} \\
& \left.+(82944 \zeta(3)-4480) C_{F}^{2}+78208 T_{F}^{2} N_{f}^{2}\right] a^{3}+O\left(a^{4}\right)
\end{aligned}
$$

in four dimensions. We adopt the same convention for the renormalization group functions as for the renormalization constants in that the scheme the variables correspond to is indicated on the left hand side. Restricting to $S U(3)$ and the Landau gauge we have

$$
\begin{aligned}
\left.\gamma_{\bar{\psi} \gamma^{\mu} D^{\nu} \psi}^{\mathrm{RI}^{\prime}}(a)\right|_{\alpha=0} ^{S U(3)}= & \frac{32}{9} a-\frac{4}{243}\left[378 N_{f}-6005\right] a^{2} \\
+\frac{8}{6561}[ & 10998 N_{f}^{2}-6318 \zeta(3) N_{f}-467148 N_{f} \\
& -524313 \zeta(3)+3691019] a^{3}+O\left(a^{4}\right) .
\end{aligned}
$$

As an aid to the lattice matching procedure we note that the finite parts of the various components in the two schemes after renormalization are

$$
\begin{aligned}
\left.\sum_{\bar{\psi} \gamma^{\mu} D^{\nu} \psi}^{(1) \overline{\mathrm{MS}} \text { finite }}(p)\right|_{p^{2}=\mu^{2}}=1 & -\frac{31}{9} C_{F} a-\left[\left(\frac{9487}{324}-\frac{5}{3} \alpha-\frac{1}{8} \alpha^{2}-8 \zeta(3)+2 \zeta(3) \alpha\right) C_{F} C_{A}\right. \\
& \left.-\frac{2101}{162} T_{F} N_{f} C_{F}+\left(\frac{1}{2} \alpha^{2}-\frac{3}{2} \alpha-\frac{8195}{648}+8 \zeta(3)\right) C_{F}^{2}\right] a^{2} \\
- & {\left[\left(\frac{635}{216} \alpha-\frac{452579}{2187}-\frac{976}{81} \zeta(3)-\frac{4}{9} \zeta(3) \alpha-\frac{64}{3} \zeta(4)\right) T_{F} N_{f} C_{F} C_{A}\right.} \\
& +\left(\frac{85}{9} \alpha-\frac{61322}{2187}+\frac{272}{9} \zeta(3)-\frac{64}{9} \zeta(3) \alpha+\frac{64}{3} \zeta(4)\right) T_{F} N_{f} C_{F}^{2} \\
& +\left(\frac{63602}{2187}+\frac{256}{81} \zeta(3)\right) T_{F}^{2} N_{f}^{2} C_{F} \\
& +\left(\frac{21026833}{69984}-\frac{953}{1728} \alpha+\frac{75}{64} \alpha^{2}+\frac{1}{48} \alpha^{3}-\left(\frac{135}{4}+\frac{5}{4} \alpha^{2}\right) \zeta(5)\right. \\
& +\left(\frac{1}{3} \alpha^{3}+2 \alpha^{2}+\frac{167}{36} \alpha-\frac{3230}{81}\right) \zeta(3) \\
& \left.+\left(\frac{719}{48}-\frac{3}{8} \alpha-\frac{3}{16} \alpha^{2}\right) \zeta(4)\right) C_{F} C_{A}^{2}
\end{aligned}
$$




$$
\begin{gathered}
+\left(\frac{5}{8} \alpha^{3}-\frac{41}{72} \alpha^{2}-\frac{979}{27} \alpha-\frac{605431}{4374}+\left(\frac{440}{3}-\frac{40}{3} \alpha\right) \zeta(5)\right. \\
\left.-\left(\frac{439}{9}-\frac{53}{2} \alpha-\frac{1}{6} \alpha^{2}+\alpha^{3}\right) \zeta(3)-38 \zeta(4)\right) C_{F}^{2} C_{A} \\
+\left(\left(\frac{7964}{81}-\frac{164}{9} \alpha-\frac{16}{3} \alpha^{2}+\frac{2}{3} \alpha^{3}\right) \zeta(3)+\frac{64}{3} \zeta(4)-\frac{400}{3} \zeta(5)\right. \\
\left.\left.\quad-\frac{2935}{5832}+\frac{821}{36} \alpha+\frac{79}{36} \alpha^{2}-\frac{1}{4} \alpha^{3}\right) C_{F}^{3}\right] a^{3}+O\left(a^{4}\right)
\end{gathered}
$$

$$
\begin{aligned}
\left.\sum_{\bar{\psi} \gamma^{\mu} D^{\nu} \psi}^{(2) \overline{\mathrm{MS}} \text { finite }}(p)\right|_{p^{2}=\mu^{2}}= & -\left(\frac{2}{3}+2 \alpha\right) C_{F} a \\
- & {\left[\left(\frac{284}{9}+\frac{29}{3} \alpha+2 \alpha^{2}-6 \zeta(3)-2 \zeta(3) \alpha\right) C_{F} C_{A}\right.} \\
& \left.-\frac{20}{3} T_{F} N_{f} C_{F}+\left(\alpha^{2}-\frac{35}{9} \alpha-\frac{74}{3}+16 \zeta(3)\right) C_{F}^{2}\right] a^{2} \\
- & \left(\frac{184}{3} \zeta(3)+\frac{136}{9} \zeta(3) \alpha-\frac{2267}{54} \alpha-\frac{87980}{243}\right) T_{F} N_{f} C_{F} C_{A} \\
& +\left(\frac{1399}{81} \alpha+\frac{4913}{27}-\frac{1088}{9} \zeta(3)\right) T_{F} N_{f} C_{F}^{2}+\frac{9680}{243} T_{F}^{2} N_{f}^{2} C_{F} \\
& +\left(\frac{2762093}{3888}+\frac{28855}{216} \alpha+\frac{431}{16} \alpha^{2}+\frac{37}{8} \alpha^{3}+\left(\frac{445}{3}+5 \alpha\right) \zeta(5)\right. \\
& \left.-\left(\frac{23}{4} \alpha^{2}+\frac{1165}{18} \alpha+\frac{1617}{4}\right) \zeta(3)\right) C_{F} C_{A}^{2} \\
& \left(\alpha^{3}+\frac{55}{36} \alpha^{2}-\frac{4790}{81} \alpha-\frac{198059}{324}-\left(\frac{1480}{3}-\frac{40}{3} \alpha\right) \zeta(5)\right. \\
& \left.+\left(\frac{7450}{9}+\frac{131}{9} \alpha-5 \alpha^{2}\right) \zeta(3)\right) C_{F}^{2} C_{A} \\
+ & \left(\frac{1760}{3} \zeta(5)-\left(\frac{4232}{9}+8 \alpha\right) \zeta(3)-\frac{29483}{972}\right. \\
& \left.\left.+\frac{3281}{324} \alpha+\frac{79}{18} \alpha^{2}-\frac{1}{2} \alpha^{3}\right) C_{F}^{3}\right] a^{3}+O\left(a^{4}\right)
\end{aligned}
$$

and

$$
\begin{aligned}
\left.\sum_{\bar{\psi} \gamma^{\mu} D^{\nu} \psi}^{(2) \mathrm{RI}^{\prime} \text { finite }}(p)\right|_{p^{2}=\mu^{2}}= & -\left(\frac{2}{3}+2 \alpha\right) C_{F} a \\
- & {\left[\left(\frac{284}{9}+\frac{271}{18} \alpha+3 \alpha^{2}+\frac{1}{2} \alpha^{3}-6 \zeta(3)-2 \zeta(3) \alpha\right) C_{F} C_{A}\right.} \\
& -\left(\frac{20}{3}+\frac{40}{9} \alpha\right) T_{F} N_{f} C_{F} \\
& \left.+\left(\alpha^{2}+3 \alpha-\frac{604}{27}+16 \zeta(3)\right) C_{F}^{2}\right] a^{2} \\
- & {\left[\frac{184}{3} \zeta(3)+\frac{32}{9} \zeta(3) \alpha-\frac{20}{9} \alpha^{3}\right.} \\
& \left.-\frac{40}{3} \alpha^{2}-\frac{9470}{81} \alpha-\frac{87980}{243}\right) T_{F} N_{f} C_{F} C_{A} \\
& -\left(\frac{40}{9} \alpha^{2}+52 \alpha-\frac{36536}{243}+\frac{1088}{9} \zeta(3)-32 \zeta(3) \alpha\right) T_{F} N_{f} C_{F}^{2} \\
& +\left(\frac{9680}{243}+\frac{800}{81} \alpha\right) T_{F}^{2} N_{f}^{2} C_{F}
\end{aligned}
$$




$$
\begin{aligned}
& +\left(\frac{2762093}{3888}+\frac{289991}{1296} \alpha+\frac{569}{12} \alpha^{2}+\frac{127}{9} \alpha^{3}+\frac{19}{8} \alpha^{4}+\frac{1}{4} \alpha^{5}\right. \\
& +\left(\frac{1}{2} \alpha^{3}+\frac{11}{4} \alpha^{2}+\frac{685}{9} \alpha+\frac{1617}{4}\right) \zeta(3) \\
& \left.\quad+\left(\frac{445}{3}+5 \alpha\right) \zeta(5)\right) C_{F} C_{A}^{2} \\
& +\left(\frac{1}{2} \alpha^{4}+\frac{5}{2} \alpha^{3}+\frac{107}{9} \alpha^{2}+\frac{1429}{36} \alpha-\frac{469555}{972}\right. \\
& \left.\quad+\left(\frac{7216}{9}-7 \alpha-\alpha^{2}\right) \zeta(3)-\left(\frac{1480}{3}-\frac{40}{3} \alpha\right) \zeta(5)\right) C_{F}^{2} C_{A} \\
& +\left(\frac{1760}{3} \zeta(5)-\left(\frac{3688}{9}-8 \alpha\right) \zeta(3)-\frac{56287}{486}\right. \\
& \left.\left.\quad-\frac{35}{6} \alpha+\frac{31}{6} \alpha^{2}+\frac{1}{2} \alpha^{3}\right) C_{F}^{3}\right] a^{3}+O\left(a^{4}\right) .
\end{aligned}
$$

These imply

$$
\begin{aligned}
\left.\Sigma_{\bar{\psi} \gamma^{\mu} D^{\nu} \psi}^{(1) \overline{\mathrm{MS}} \text { finite }}(p)\right|_{p^{2}=\mu^{2}} ^{S U(3), \alpha=0}=1 & -\frac{124}{27} a-\left(\frac{68993}{729}-\frac{160}{9} \zeta(3)-\frac{2101}{243} N_{f}\right) a^{2} \\
- & {\left[\frac{451293899}{157464}-\frac{1105768}{2187} \zeta(3)+\frac{8959}{324} \zeta(4)+\frac{4955}{81} \zeta(5)\right.} \\
& +\left(\frac{224}{81} \zeta(3)-\frac{640}{27} \zeta(4)-\frac{8636998}{19683}\right) N_{f} \\
& \left.+\left(\frac{63602}{6561}+\frac{256}{243} \zeta(3)\right) N_{f}^{2}\right] a^{3}+O\left(a^{4}\right)
\end{aligned}
$$

and

$$
\begin{aligned}
\left.\sum_{\bar{\psi} \gamma^{\mu} D^{\nu} \psi}^{(2) \overline{\mathrm{MS}} \text { finite }}(p)\right|_{p^{2}=\mu^{2}} ^{S U(3), \alpha=0}= & -\frac{8}{9} a-\left(\frac{2224}{27}+\frac{40}{9} \zeta(3)-\frac{40}{9} N_{f}\right) a^{2} \\
& -\left[\frac{136281133}{26244}-\frac{376841}{243} \zeta(3)+\frac{43700}{81} \zeta(5)\right. \\
& \left.+\left(\frac{1232}{81} \zeta(3)-\frac{15184}{27}\right) N_{f}+\frac{9680}{729} N_{f}^{2}\right] a^{3} \\
& +O\left(a^{4}\right) .
\end{aligned}
$$

\section{Second moment of transversity operator.}

The renormalization of the transversity operator $\bar{\psi} \sigma^{\mu \nu} D^{\rho} \psi$ is similar to that for $\bar{\psi} \gamma^{\mu} D^{\nu} \psi$ aside from the decomposition of the corresponding Green's function. The specific operator we are interested in is

$$
\begin{aligned}
\mathcal{S} \bar{\psi} \sigma^{\mu \nu} D^{\rho} \psi= & \bar{\psi} \sigma^{\mu \nu} D^{\rho} \psi+\bar{\psi} \sigma^{\mu \rho} D^{\nu} \psi-\frac{2}{(d-1)} \eta^{\nu \rho} \bar{\psi} \sigma^{\mu \lambda} D_{\lambda} \psi \\
& +\frac{1}{(d-1)}\left(\eta^{\mu \nu} \bar{\psi} \sigma^{\rho \lambda} D_{\lambda} \psi+\eta^{\mu \rho} \bar{\psi} \sigma^{\nu \lambda} D_{\lambda} \psi\right)
\end{aligned}
$$

which is symmetric with respect to the indices $\nu$ and $\rho$ and satisfies the traceless conditions, 37.

$$
\mathcal{S} \bar{\psi} \sigma^{\mu \nu} D_{\mu} \psi=\mathcal{S} \bar{\psi} \sigma^{\mu \nu} D_{\nu} \psi=0
$$


The Lorentz decomposition of the particular Green's function we will renormalize is

$$
\begin{aligned}
G_{\bar{\psi} \sigma^{\mu \nu} D^{\rho} \psi}^{\mu \nu \rho}(p)= & \left\langle\psi(p)\left[\mathcal{S} \bar{\psi} \sigma^{\mu \nu} D^{\rho} \psi\right](0) \bar{\psi}(-p)\right\rangle \\
= & \Sigma_{\bar{\psi} \sigma^{\mu \nu} D^{\rho} \psi}^{(1)}(p)\left(\sigma^{\mu \nu} p^{\rho}+\sigma^{\mu \rho} p^{\nu}-\frac{(d+2)}{p^{2}} \sigma^{\mu \lambda} p^{\nu} p^{\rho} p_{\lambda}+\eta^{\nu \rho} \sigma^{\mu \lambda} p_{\lambda}\right) \\
& +\Sigma_{\bar{\psi} \sigma^{\mu \nu} D^{\rho} \psi}^{(2)}(p)\left(\eta^{\mu \nu} \sigma^{\rho \lambda} p_{\lambda}+\eta^{\mu \rho} \sigma^{\nu \lambda} p_{\lambda}+\frac{(d-1)(d+2)}{p^{2}} \sigma^{\mu \lambda} p^{\nu} p^{\rho} p_{\lambda}\right. \\
& \left.\quad-(d+1) \eta^{\nu \rho} \sigma^{\mu \lambda} p_{\lambda}\right) \\
& +\Sigma_{\bar{\psi} \sigma^{\mu \nu} D^{\rho} \psi}^{(3)}(p)\left(\sigma^{\nu \lambda} p^{\mu} p^{\rho} p_{\lambda}+\sigma^{\rho \lambda} p^{\mu} p^{\nu} p_{\lambda}+d \sigma^{\mu \lambda} p^{\nu} p^{\rho} p_{\lambda}-\eta^{\nu \rho} \sigma^{\mu \lambda} p_{\lambda} p^{2}\right)
\end{aligned}
$$

where the components are determined through

$$
\begin{aligned}
& \Sigma_{\bar{\psi} \sigma^{\mu \nu} D^{\rho} \psi}^{(1)}(p)=-\frac{1}{8(d-1)(d-2)} \operatorname{tr}\left[\left(\sigma_{\mu \nu} p_{\rho}+\sigma_{\mu \rho} p_{\nu}-\frac{(d+2)}{p^{2}} \sigma_{\mu \lambda} p_{\nu} p_{\rho} p^{\lambda}\right.\right. \\
& \left.\left.+\eta_{\nu \rho} \sigma_{\mu \lambda} p^{\lambda}\right) G_{\bar{\psi} \sigma^{\mu \nu} D^{\rho} \psi}^{\mu \nu \rho}(p)\right] \\
& -\frac{1}{8(d-1)(d-2) p^{2}} \operatorname{tr}\left[\left(\sigma_{\nu \lambda} p_{\mu} p_{\rho} p^{\lambda}+\sigma_{\rho \lambda} p_{\mu} p_{\nu} p^{\lambda}\right.\right. \\
& \left.\left.+d \sigma_{\mu \lambda} p_{\nu} p_{\rho} p^{\lambda}-\eta_{\nu \rho} \sigma_{\mu \lambda} p^{\lambda} p^{2}\right) G_{\bar{\psi} \sigma^{\mu \nu} D^{\rho} \psi}^{\mu \nu \rho}(p)\right] \\
& \Sigma_{\bar{\psi} \sigma^{\mu \nu} D^{\rho} \psi}^{(2)}(p)=-\frac{\left(d^{2}-d+2\right)}{8(d-1)\left(d^{2}-1\right)\left(d^{2}-4\right)} \operatorname{tr}\left[\left(\eta_{\mu \nu} \sigma_{\rho \lambda} p^{\lambda}+\eta_{\mu \rho} \sigma_{\nu \lambda} p^{\lambda}-(d+1) \eta_{\nu \rho} \sigma_{\mu \lambda} p^{\lambda}\right.\right. \\
& \left.\left.+\frac{(d-1)(d+2)}{p^{2}} \sigma_{\mu \lambda} p_{\nu} p_{\rho} p^{\lambda}\right) G_{\bar{\psi} \sigma^{\mu \nu} D^{\rho} \psi}^{\mu \nu \rho}(p)\right] \\
& +\frac{1}{8(d+1)(d-2) p^{2}} \operatorname{tr}\left[\left(\sigma_{\nu \lambda} p_{\mu} p_{\rho} p^{\lambda}+\sigma_{\rho \lambda} p_{\mu} p_{\nu} p^{\lambda}\right.\right. \\
& \left.\left.+d \sigma_{\mu \lambda} p_{\nu} p_{\rho} p^{\lambda}-\eta_{\nu \rho} \sigma_{\mu \lambda} p^{\lambda} p^{2}\right) G_{\bar{\psi} \sigma^{\mu \nu} D^{\rho} \psi}^{\mu \nu \rho}(p)\right] \\
& \Sigma_{\bar{\psi} \sigma^{\mu \nu} D^{\rho} \psi}^{(3)}(p)=-\frac{1}{8(d-1)(d-2)} \operatorname{tr}\left[\left(\sigma_{\mu \nu} p_{\rho}+\sigma_{\mu \rho} p_{\nu}-\frac{(d+2)}{p^{2}} \sigma_{\mu \lambda} p_{\nu} p_{\rho} p^{\lambda}\right.\right. \\
& \left.\left.+\eta_{\nu \rho} \sigma_{\mu \lambda} p^{\lambda}\right) G_{\bar{\psi} \sigma^{\mu \nu} D^{\rho} \psi}^{\mu \nu \rho}(p)\right] \\
& +\frac{1}{8(d+1)(d-2)} \operatorname{tr}\left[\left(\eta_{\mu \nu} \sigma_{\rho \lambda} p^{\lambda}+\eta_{\mu \rho} \sigma_{\nu \lambda} p^{\lambda}-(d+1) \eta_{\nu \rho} \sigma_{\mu \lambda} p^{\lambda}\right.\right. \\
& \left.\left.+\frac{(d-1)(d+2)}{p^{2}} \sigma_{\mu \lambda} p_{\nu} p_{\rho} p^{\lambda}\right) G_{\bar{\psi} \sigma^{\mu \nu} D^{\rho} \psi}^{\mu \nu \rho}(p)\right] \\
& -\frac{(d+2)}{8(d+1)(d-2) p^{2}} \operatorname{tr}\left[\left(\sigma_{\nu \lambda} p_{\mu} p_{\rho} p^{\lambda}+\sigma_{\rho \lambda} p_{\mu} p_{\nu} p^{\lambda}\right.\right. \\
& \left.\left.+d \sigma_{\mu \lambda} p_{\nu} p_{\rho} p^{\lambda}-\eta_{\nu \rho} \sigma_{\mu \lambda} p^{\lambda} p^{2}\right) G_{\bar{\psi} \sigma^{\mu \nu} D^{\rho} \psi}^{\mu \nu}(p)\right] .
\end{aligned}
$$

Using the same programmes which determined the renormalization of $\bar{\psi} \gamma^{\mu} D^{\nu} \psi$ but instead including the Feynman rule for the transversity operator, we find after renormalizing the Green's function in the $\overline{\mathrm{MS}}$ scheme that

$$
\begin{aligned}
Z_{\overline{\bar{\psi} \sigma^{\mu \nu} D^{\rho} \psi}}^{\overline{\mathrm{MS}}=} & 1+\frac{3}{\epsilon} C_{F} a \\
& +\left[\left(4 T_{F} N_{f}+9 C_{F}-11 C_{A}\right) \frac{1}{2 \epsilon^{2}}+\left(35 C_{A}-9 C_{F}-12 T_{F} N_{f}\right) \frac{1}{4 \epsilon}\right] C_{F} a^{2} \\
& +\left[\left(242 C_{A}^{2}-297 C_{A} C_{F}-176 C_{A} T_{F} N_{f}+81 C_{F}^{2}+108 C_{F} T_{F} N_{f}+32 T_{F}^{2} N_{f}^{2}\right) \frac{1}{18 \epsilon^{3}}\right.
\end{aligned}
$$




$$
\begin{aligned}
& +\quad\left(1143 C_{A} C_{F}-1178 C_{A}^{2}+784 C_{A} T_{F} N_{f}\right. \\
& \left.\quad-243 C_{F}^{2}-252 C_{F} T_{F} N_{f}-96 T_{F}^{2} N_{f}^{2}\right) \frac{1}{36 \epsilon^{2}} \\
& +\quad\left(12553 C_{A}^{2}-7479 C_{A} C_{F}-(5184 \zeta(3)+4168) C_{A} T_{F} N_{f}+1782 C_{F}^{2}\right. \\
& \left.\left.\quad+(5184 \zeta(3)-3240) C_{F} T_{F} N_{f}-368 T_{F}^{2} N_{f}^{2}\right) \frac{1}{324 \epsilon}\right] C_{F} a^{3}+O\left(a^{4}\right)
\end{aligned}
$$

and with the $\mathrm{RI}^{\prime}$ scheme definition

$$
\left.\lim _{\epsilon \rightarrow 0}\left[Z_{\psi}^{\mathrm{RI}^{\prime}} Z_{\bar{\psi} \sigma^{\mu \nu} D^{\rho} \psi}^{\mathrm{RI}_{\psi}^{\prime}} \Sigma_{\bar{\psi} \sigma^{\mu \nu} D^{\rho} \psi}^{(1)}(p)\right]\right|_{p^{2}=\mu^{2}}=1
$$

we have

$$
\begin{aligned}
& Z_{\bar{\psi} \sigma^{\mu \nu} D^{\rho} \psi}^{\mathrm{RI}^{\prime}}=1+\left[\frac{3}{\epsilon}+\frac{1}{2}(3 \alpha+7)\right] C_{F} a \\
& +\left[\left(4 T_{F} N_{f}+9 C_{F}-11 C_{A}\right) \frac{1}{2 \epsilon^{2}}+\left(35 C_{A}+(33+18 \alpha) C_{F}-12 T_{F} N_{f}\right) \frac{1}{4 \epsilon}\right. \\
& +\frac{1}{24}\left(\left(9 \alpha^{3}+54 \alpha^{2}-36 \zeta(3) \alpha+271 \alpha-324 \zeta(3)+1189\right) C_{A}\right. \\
& \left.\left.+\left(36 \alpha^{2}+108 \alpha+288 \zeta(3)-165\right) C_{F}-(80 \alpha+468) T_{F} N_{f}\right)\right] a^{2} \\
& +\left[\left(242 C_{A}^{2}-297 C_{A} C_{F}-176 C_{A} T_{F} N_{f}+81 C_{F}^{2}+108 C_{F} T_{F} N_{f}+32 T_{F}^{2} N_{f}^{2}\right) \frac{1}{18 \epsilon^{3}}\right. \\
& +\left((450-297 \alpha) C_{A} C_{F}-1178 C_{A}^{2}+784 C_{A} T_{F} N_{f}+(243 \alpha+324) C_{F}^{2}\right. \\
& \left.+108 \alpha C_{F} T_{F} N_{f}-96 T_{F}^{2} N_{f}^{2}\right) \frac{1}{36 \epsilon^{2}} \\
& +\left(\left(729 \alpha^{3}+4374 \alpha^{2}-2916 \zeta(3) \alpha+30456 \alpha-26244 \zeta(3)+101196\right) C_{A} C_{F}\right. \\
& +25106 C_{A}^{2}-(10368 \zeta(3)+8336) C_{A} T_{F} N_{f} \\
& +\left(2916 \alpha^{2}+6561 \alpha-14904+23328 \zeta(3)\right) C_{F}^{2}-736 T_{F}^{2} N_{f}^{2} \\
& \left.+(10368 \zeta(3)-51192-9396 \alpha) C_{F} T_{F} N_{f}\right) \frac{1}{648 \epsilon} \\
& +\frac{1}{5184}\left(\left(972 \alpha^{5}+9234 \alpha^{4}-1944 \zeta(3) \alpha^{3}+54864 \alpha^{3}-10692 \zeta(3) \alpha^{2}\right.\right. \\
& +184356 \alpha^{2}-282312 \zeta(3) \alpha+19440 \zeta(5) \alpha+874185 \alpha \\
& -1644228 \zeta(3)+447120 \zeta(5)+3930425) C_{A}^{2} \\
& +\left(3888 \alpha^{4}+24300 \alpha^{3}+3888 \zeta(3) \alpha^{2}+116748 \alpha^{2}\right. \\
& -34992 \zeta(3) \alpha+51840 \zeta(5) \alpha+375732 \alpha+1260576 \zeta(3) \\
& \text { - 466560弓(5) - 511188) } C_{A} C_{F} \\
& \text { - }\left(8640 \alpha^{3}+51840 \alpha^{2}-13824 \zeta(3) \alpha+454560 \alpha\right. \\
& -124992 \zeta(3)+124416 \zeta(4)+2438256) C_{A} T_{F} N_{f} \\
& +(38400 \alpha+18432 \zeta(3)+323200) T_{F}^{2} N_{f}^{2} \\
& \text { - }\left(34560 \alpha^{2}-82944 \zeta(3) \alpha+295920 \alpha-82944 \zeta(3)\right. \\
& \text { - } 124416 \zeta(4)+620592) C_{F} T_{F} N_{f} \\
& +\left(7776 \alpha^{3}-31104 \zeta(3) \alpha^{2}+54432 \alpha^{2}+32076 \alpha+51840 \zeta(3)\right.
\end{aligned}
$$




$$
\left.\left.+207360 \zeta(5)-266868) C_{F}^{2}\right)\right] C_{F} a^{3}+O\left(a^{4}\right)
$$

Consequently, the renormalization group function

$$
\begin{aligned}
\gamma_{\bar{\psi} \sigma^{\mu \nu} D^{\rho} \psi}^{\overline{\mathrm{MS}}}(a)= & 3 C_{F} a+\frac{C_{F}}{2}\left[35 C_{A}-9 C_{F}-12 T_{F} N_{f}\right] a^{2} \\
& +\frac{C_{F}}{108}\left[12553 C_{A}^{2}-7479 C_{A} C_{F}-(5184 \zeta(3)+4168) C_{A} T_{F} N_{f}\right. \\
& \left.\quad+1782 C_{F}^{2}+(5184 \zeta(3)-3240) C_{F} T_{F} N_{f}-368 T_{F}^{2} N_{f}^{2}\right] a^{3} \\
& +O\left(a^{4}\right)
\end{aligned}
$$

has emerged as being independent of $\alpha$ as expected. The two loop part of (3.8) agrees with the known results, 38, 39, 40, 37, 41, when they are restricted to the second moment. A second check on the result is that the double and triple poles in $\epsilon$ at three loops in the renormalization constant have correctly emerged. Also, in four dimensions

$$
\begin{aligned}
\gamma_{\bar{\psi} \sigma^{\mu \nu} D^{\rho} \psi}^{\mathrm{RI}^{\prime}}(a)=3 C_{F} a+ & \frac{C_{F}}{12}\left[\left(9 \alpha^{2}+27 \alpha+364\right) C_{A}-54 C_{F}-128 T_{F} N_{f}\right] a^{2} \\
+\frac{C_{F}}{432}[ & \left(162 \alpha^{4}+1377 \alpha^{3}-324 \zeta(3) \alpha^{2}+6633 \alpha^{2}-3348 \zeta(3) \alpha\right. \\
& +21366 \alpha-42768 \zeta(3)+224296) C_{A}^{2} \\
& +\left(162 \alpha^{3}+324 \alpha^{2}-1674 \alpha+38016 \zeta(3)-71100\right) C_{A} C_{F} \\
& -\left(1440 \alpha^{2}-864 \zeta(3) \alpha+7416 \alpha+5184 \zeta(3)+145600\right) C_{A} T_{F} N_{f} \\
& +(432 \alpha+6912 \zeta(3)-4032) C_{F} T_{F} N_{f} \\
& \left.+7128 C_{F}^{2}+20992 T_{F}^{2} N_{f}^{2}\right] a^{3}+O\left(a^{4}\right) .
\end{aligned}
$$

Thus, for $S U(3)$ and the Landau gauge we have

$$
\begin{aligned}
\left.\gamma_{\overline{\bar{\psi}} \sigma^{\mu \nu} D^{\rho} \psi}^{\overline{\mathrm{MS}}}(a)\right|_{\alpha=0} ^{S U(3)}= & 4 a-2\left[2 N_{f}-31\right] a^{2} \\
& -\frac{1}{81}\left[92 N_{f}^{2}+4320 \zeta(3) N_{f}+8412 N_{f}-86229\right] a^{3}+O\left(a^{4}\right)
\end{aligned}
$$

and

$$
\begin{aligned}
\left.\gamma_{\bar{\psi} \sigma^{\mu \nu} D^{\rho} \psi}^{\mathrm{RI}^{\prime}}(a)\right|_{\alpha=0} ^{S U(3)}= & 4 a-\frac{4}{9}\left[16 N_{f}-255\right] a^{2} \\
& +\frac{2}{81}\left[656 N_{f}^{2}-(396 \zeta(3)+27636) N_{f}-29106 \zeta(3)+218367\right] a^{3} \\
& +O\left(a^{4}\right)
\end{aligned}
$$

which are the main results of this article. To assist with the lattice matching procedure we record that the finite parts of the components in both the $\overline{\mathrm{MS}}$ and $\mathrm{RI}^{\prime}$ schemes after renormalization are

$$
\begin{aligned}
\left.\sum_{\bar{\psi} \sigma^{\mu \nu} D^{\rho} \psi}^{(1) \overline{\mathrm{MS}} \text { finite }}(p)\right|_{p^{2}=\mu^{2}}=1 & -\left(\frac{7}{2}+\frac{\alpha}{2}\right) C_{F} a \\
- & {\left[\left(\frac{943}{24}+\frac{3}{4} \alpha+\frac{3}{8} \alpha^{2}-\frac{21}{2} \zeta(3)+\frac{3}{2} \zeta(3) \alpha\right) C_{F} C_{A}-16 T_{F} N_{f} C_{F}\right.} \\
& \left.-\left(\frac{37}{2}+\frac{5}{2} \alpha-\frac{3}{4} \alpha^{2}-12 \zeta(3)\right) C_{F}^{2}\right] a^{2}
\end{aligned}
$$




$$
\begin{aligned}
& -\left[\left(\frac{478}{27}+\frac{47}{3} \alpha-8 \zeta(3) \alpha+24 \zeta(4)\right) T_{F} N_{f} C_{F}^{2}\right. \\
& +\left(\frac{1160}{27}+\frac{32}{9} \zeta(3)\right) T_{F}^{2} N_{f}^{2} C_{F} \\
& +\left(\left(\frac{61}{9}+\frac{10}{3} \alpha\right) \zeta(3)-24 \zeta(4)-\frac{104843}{324}-\frac{68}{9} \alpha\right) T_{F} N_{f} C_{F} C_{A} \\
& +\left(\frac{2656369}{5184}+\frac{18379}{576} \alpha+\frac{253}{32} \alpha^{2}+\frac{113}{96} \alpha^{3}\right. \\
& -\left(\frac{26839}{144}+\frac{131}{12} \alpha-\frac{9}{16} \alpha^{2}-\frac{1}{3} \alpha^{3}\right) \zeta(3) \\
& +\left(\frac{69}{16}-\frac{3}{8} \alpha-\frac{3}{16} \alpha^{2}\right) \zeta(4) \\
& \left.+\left(45+\frac{5}{4} \alpha-\frac{5}{4} \alpha^{2}\right) \zeta(5)\right) C_{F} C_{A}^{2} \\
& +\left(\left(\frac{1699}{6}+\frac{131}{4} \alpha-\frac{3}{4} \alpha^{2}-\alpha^{3}\right) \zeta(3)-(70+10 \alpha) \zeta(5)\right. \\
& \left.-6 \zeta(4)-\frac{158969}{432}-\frac{2657}{48} \alpha-\frac{5}{8} \alpha^{2}+\frac{7}{8} \alpha^{3}\right) C_{F}^{2} C_{A} \\
& +\left(40 \zeta(5)-\left(74+24 \alpha+6 \alpha^{2}-\frac{2}{3} \alpha^{3}\right) \zeta(3)\right. \\
& \left.\left.+\frac{521}{12}+\frac{59}{2} \alpha+\frac{33}{8} \alpha^{2}-\frac{3}{8} \alpha^{3}\right) C_{F}^{3}\right] a^{3}+O\left(a^{4}\right) \\
& \left.\Sigma_{\bar{\psi} \sigma^{\mu \nu} D^{\rho} \psi}^{(2) \overline{\mathrm{MS}} \text { finite }}(p)\right|_{p^{2}=\mu^{2}}=\left.\frac{1}{3} \Sigma_{\bar{\psi} \sigma^{\mu \nu} D^{\rho} \psi}^{(1) \overline{\mathrm{MS}} \text { finite }}(p)\right|_{p^{2}=\mu^{2}}+O\left(a^{4}\right)
\end{aligned}
$$

and

$$
\left.\Sigma_{\bar{\psi} \sigma^{\mu \nu} D^{\rho} \psi}^{(3) \overline{\mathrm{MS}} \text { finite }}(p)\right|_{p^{2}=\mu^{2}}=\left.\Sigma_{\bar{\psi} \sigma^{\mu \nu} D^{\rho} \psi}^{(3) \mathrm{RI}^{\prime} \text { finite }}(p)\right|_{p^{2}=\mu^{2}}=O\left(a^{4}\right)
$$

to three loops. Hence,

$$
\begin{aligned}
\left.\Sigma_{\bar{\psi} \sigma^{\mu \nu} D^{\rho} \psi}^{(1) \overline{\mathrm{MS}} \text { finite }}(p)\right|_{p^{2}=\mu^{2}} ^{S U(3), \alpha=0}=1 & -\frac{14}{3} a-\left(\frac{2237}{18}-\frac{62}{3} \zeta(3)-\frac{32}{3} N_{f}\right) a^{2} \\
- & \frac{1852993}{432}-\frac{97391}{108} \zeta(3)+\frac{79}{4} \zeta(4)+\frac{7060}{27} \zeta(5) \\
& +\left(\frac{122}{9} \zeta(3)-\frac{80}{3} \zeta(4)-\frac{306881}{486}\right) N_{f} \\
& \left.+\left(\frac{1160}{81}+\frac{32}{27} \zeta(3)\right) N_{f}^{2}\right] a^{3}+O\left(a^{4}\right) .
\end{aligned}
$$

\section{Conversion functions.}

As in 32 we have checked our expressions by also constructing the conversion functions $C_{\mathcal{O}}(a, \alpha)$ for each of the operators explicitly. These functions allow one to move from one scheme to another and are defined by, 42,

$$
C_{\mathcal{O}}(a, \alpha)=\frac{Z_{\mathcal{O}}^{\mathrm{RI}^{\prime}}}{Z_{\overline{\mathcal{O}}}^{\overline{\mathrm{MS}}}} .
$$

The renormalization group functions are then related by

$$
\gamma_{\mathcal{O}}^{\mathrm{RI}^{\prime}}\left(a_{\mathrm{RI}^{\prime}}\right)=\gamma_{\mathcal{O}}^{\overline{\mathrm{MS}}}\left(a_{\overline{\mathrm{MS}}}\right)-\beta\left(a_{\overline{\mathrm{MS}}}\right) \frac{\partial}{\partial a_{\overline{\mathrm{MS}}}} \ln C_{\mathcal{O}}\left(a_{\overline{\mathrm{MS}}}, \alpha \overline{\mathrm{MS}}\right)
$$




$$
-\alpha_{\overline{\mathrm{MS}}} \gamma_{\alpha}^{\overline{\mathrm{MS}}}\left(a_{\overline{\mathrm{MS}}}\right) \frac{\partial}{\partial \alpha_{\overline{\mathrm{MS}}}} \ln C_{\mathcal{O}}\left(a_{\overline{\mathrm{MS}}}, \alpha_{\overline{\mathrm{MS}}}\right)
$$

where we have indicated the scheme of the variables explicitly. Being careful to first convert the variables to the same reference scheme, which we will take to be the $\overline{\mathrm{MS}}$ scheme, we have from the various renormalization constants,

$$
\begin{aligned}
C_{\bar{\psi} \gamma^{\mu} D^{\nu} \psi}(a, \alpha)=1+ & (9 \alpha+31) \frac{C_{F} a}{9} \\
+ & {\left[\left(162 \alpha^{2}-162 \zeta(3) \alpha+783 \alpha-1782 \zeta(3)+6404\right) C_{A}\right.} \\
& \left.+\quad\left(81 \alpha^{2}+315 \alpha+1296 \zeta(3)-228\right) C_{F}-2668 T_{F} N_{f}\right] \frac{C_{F} a^{2}}{162} \\
+ & {\left[\left(161838 \alpha^{3}-201204 \zeta(3) \alpha^{2}+942597 \alpha^{2}-2124792 \zeta(3) \alpha\right.\right.} \\
& +174960 \zeta(5) \alpha+4796982 \alpha-11944044 \zeta(3)+746496 \zeta(4) \\
+ & 524880 \zeta(5)+38226589) C_{A}^{2} \\
+ & \left(26244 \alpha^{3}+151632 \zeta(3) \alpha^{2}+159408 \alpha^{2}+346032 \zeta(3) \alpha\right. \\
& +466560 \zeta(5) \alpha+554904 \alpha-4914432 \zeta(3) \\
& -2239488 \zeta(4)+8864640 \zeta(5)+3993332) C_{A} C_{F} \\
+ & (528768 \zeta(3) \alpha-1469016 \alpha+369792 \zeta(3) \\
& -1492992 \zeta(4)-24752896) C_{A} T_{F} N_{f} \\
+ & \left(17496 \alpha^{3}-373248 \zeta(3) \alpha^{2}+289656 \alpha^{2}-715392 \zeta(3) \alpha\right. \\
& +879336 \alpha+10737792 \zeta(3)+1492992 \zeta(4) \\
& -9331200 \zeta(5)-3848760) C_{F}^{2} \\
- & (497664 \zeta(3) \alpha+351648 \alpha-3234816 \zeta(3) \\
& -1492992 \zeta(4)+9980032) C_{F} T_{F} N_{f} \\
+ & \left.(221184 \zeta(3)+3391744) T_{F}^{2} N_{f}^{2}\right] \frac{C_{F} a^{3}}{69984}+O\left(a^{4}\right) \\
&
\end{aligned}
$$

and

$$
\begin{aligned}
C_{\bar{\psi} \sigma^{\mu \nu} D^{\rho} \psi}(a, \alpha)=1+ & (3 \alpha+7) \frac{C_{F} a}{2} \\
+ & {\left[\left(36 \alpha^{2}-36 \zeta(3) \alpha+174 \alpha-324 \zeta(3)+1189\right) C_{A}\right.} \\
& \left.+\left(36 \alpha^{2}+108 \alpha+288 \zeta(3)-165\right) C_{F}-486 T_{F} N_{f}\right] \frac{C_{F} a^{2}}{24} \\
+ & {\left[\left(17982 \alpha^{3}-22356 \zeta(3) \alpha^{2}+104733 \alpha^{2}-238032 \zeta(3) \alpha+19440 \zeta(5) \alpha\right.\right.} \\
& +523602 \alpha-1644228 \zeta(3)+447120 \zeta(5)+3930425) C_{A}^{2} \\
+ & \left(10692 \alpha^{3}+3888 \zeta(3) \alpha^{2}+63180 \alpha^{2}-34992 \zeta(3) \alpha\right. \\
& +51840 \zeta(5) \alpha+312876 \alpha+1260576 \zeta(3) \\
& -466560 \zeta(5)-511188) C_{A} C_{F} \\
+ & (58752 \zeta(3) \alpha-163224 \alpha+124992 \zeta(3) \\
& -124416 \zeta(4)-2438256) C_{A} T_{F} N_{f} \\
+ & \left(7776 \alpha^{3}-31104 \zeta(3) \alpha^{2}+54432 \alpha^{2}+32076 \alpha\right. \\
& +51840 \zeta(3)+207360 \zeta(5)-266868) C_{F}^{2}
\end{aligned}
$$




$$
\begin{aligned}
& -\quad(41472 \zeta(3) \alpha+101520 \alpha-82944 \zeta(3) \\
& \quad-124416 \zeta(4)+620592) C_{F} T_{F} N_{f} \\
& \left.+\quad(18432 \zeta(3)+323200) T_{F}^{2} N_{f}^{2}\right] \frac{C_{F} a^{3}}{5184}+O\left(a^{4}\right) .
\end{aligned}
$$

With these expressions given in terms of the $\overline{\mathrm{MS}}$ coupling constant and covariant gauge parameter, we have checked that the same RI' scheme anomalous dimensions emerge for each operator and for non-zero $\alpha$.

\section{$5 \quad$ Large $N_{f}$ critical exponent for transversity operator.}

In order to provide another independent check on our transversity results for $\overline{\mathrm{MS}}$ we have extended the leading order large $N_{f}$ calculation of the critical exponent associated with the flavour non-singlet twist-2 operator anomalous dimension as a function of $d=2 \mu$ and arbitrary moment $n$, 43, to the transversity case. In other words we consider the operator $\bar{\psi} \sigma^{\mu \nu_{1}} D^{\nu_{2}} \ldots D^{\nu_{n-1}} \psi$ which is symmetric in the indices $\left\{\nu_{i}\right\}$ and satisfies a more general traceless condition to that for $n=2$, 37. As the large $N_{f}$ critical point technique has been widely documented in 44, 45, 43. we refer interested readers to these sources and quote the main result of our computations. Following the procedure of [43], we found the $d$-dimensional critical exponent for the transversity operator is

$$
\begin{aligned}
\eta_{\bar{\psi} \sigma^{\mu \nu} D^{\rho} \psi}^{(n)}=\frac{2(\mu-1) C_{F} \eta_{1}^{\mathrm{o}}}{(2 \mu-1)(\mu-2) T_{F} N_{f}} & {\left[(\mu-1)-\frac{\mu(\mu-2)^{2}}{(\mu+n-1)(\mu+n-2)}\right.} \\
& +2 \mu[\psi(\mu+n-1)-\psi(\mu)]]+O\left(\frac{1}{N_{f}^{2}}\right)
\end{aligned}
$$

where

$$
\eta_{1}^{o}=\frac{(2 \mu-1)(\mu-2) \Gamma(2 \mu)}{4 \Gamma^{2}(\mu) \Gamma(\mu+1) \Gamma(2-\mu)}
$$

and $\psi(x)=d \ln \Gamma(x) / d x$ and $\Gamma(x)$ is the Euler gamma function. If one expands (5.1) in powers of $\epsilon, \mu=2-\epsilon$, then there is a one-to-one correspondence with the leading large $N_{f}$ coefficients of the associated anomalous dimension to all orders in the (perturbative) coupling constant. Moreover, since we have a result for arbitrary $n$ we can deduce an analytic expression for the

leading large $N_{f}$ part of the three loop term of $\gamma_{\bar{\psi} \sigma^{\mu \nu} D^{\rho} \psi}^{\overline{\mathrm{MS}}}(a)$ and check that it agrees with our result for $n=2$. In particular if we formally write

$$
\gamma_{\bar{\psi} \sigma^{\mu \nu} D^{\rho} \psi}^{(n) \overline{\mathrm{MS}}}(a)=C_{F}\left[b_{1} a+\left(b_{21} T_{F} N_{f}+b_{20}\right) a^{2}+\sum_{r=3}^{\infty} \sum_{j=0}^{r-1} b_{r j} T_{F}^{j} N_{f}^{j} a^{r}\right]
$$

where the coefficients, $\left\{b_{i j}\right\}$, are functions of $n$ and $b_{1}, b_{20}$ and $b_{21}$ are known, 38, 39, 40, 37, 41, then we find the leading order three loop coefficient is

$$
b_{32}=\frac{4}{27}\left[48 S_{3}(n)-80 S_{2}(n)-16 S_{1}(n)+\frac{3\left(17 n^{2}+17 n-8\right)}{n(n+1)}\right]
$$

where $S_{l}(n)=\sum_{i=1}^{n} 1 / i^{l}$. Setting $n=2$ we find exact agreement with the corresponding term of (3.8). Moreover, we can expand the critical exponent to four loops and deduce, using the four 
loop $\beta$-function, 46],

$$
\begin{aligned}
b_{43}=\frac{8}{81}[ & 96 S_{4}(n)-160 S_{3}(n)-32 S_{2}(n)+192 \zeta(3) S_{1}(n)-32 S_{1}(n) \\
& \left.-144 \zeta(3)+\frac{\left(131 n^{4}+262 n^{3}+211 n^{2}-16 n-48\right)}{n^{2}(n+1)^{2}}\right] .
\end{aligned}
$$

\section{Discussion.}

To conclude we have derived expressions for the three loop anomalous dimension of the second moment of the transversity operator in both the $\overline{\mathrm{MS}}$ and $\mathrm{RI}^{\prime}$ schemes. The results for the latter will be important in the extraction of lattice estimates of the associated matrix elements. Moreover, we have provided the same information for the usual twist-2 flavour non-singlet operator. Although we have had to consider various projections of the Green's function with the operator inserted in a quark two-point function it ought to be possible to extend the present calculations to derive the three loop anomalous dimensions for higher moments of the transversity operator in the $\overline{\mathrm{MS}}$ scheme. In this case since one is only interested in the divergent part and not the finite part of the Green's function, it would not require the same use of the projections considered here. This is important since the algebraic manipulations are slowed for a large number of projections and the inclusion of more covariant derivatives in the operator itself. However, since those anomalous dimensions are independent of the gauge parameter, the algebraic computations would be speeded by considering the Feynman gauge.

Acknowledgements. The author thanks Dr P.E.L. Rakow and Dr C. McNeile for valuable discussions.

\section{References.}

[1] J.P. Ralston \& D.E. Soper, Nucl. Phys. B152 (1979), 109.

[2] R.L. Jaffe \& X. Ji, Phys. Rev. Lett. 67 (1991), 552; Nucl. Phys. B375 (1992), 527.

[3] J.I. Cortes, B. Pire \& J.P. Ralston, Z. Phys. C55 (1992), 409.

[4] M. Grosse Perdekamp, A. Ogawa, K. Hasuko, S. Lange \& V. Siegle, Nucl. Phys. A711 (2002), 69c.

[5] V. Barone, A. Drago \& P.G. Ratcliffe, Phys. Rept. 359 (2002), 1; P.G. Ratcliffe, hep-ph/0211222.

[6] P.G. Ratcliffe, hep-ph/0211232.

[7] S.A. Larin, T. van Ritbergen \& J.A.M. Vermaseren, Nucl. Phys. B427 (1994), 41.

[8] S.A. Larin, P. Nogueira, T. van Ritbergen \& J.A.M. Vermaseren, Nucl. Phys. B492 (1997), 338.

[9] S. Moch \& J.A.M. Vermaseren, Acta Phys. Polon. B33 (2002), 3019.

[10] S. Moch, J.A.M. Vermaseren \& A. Vogt, Nucl. Phys. B646 (2002), 181. 
[11] J.A.M. Vermaseren, S. Moch \& A. Vogt, hep-ph/0211296

[12] D.J. Gross \& F.J. Wilczek, Phys. Rev. D9 (1974), 980.

[13] E.G. Floratos, D.A. Ross \& C.T. Sachrajda, Nucl. Phys. B129 (1977), 66; B139 (1978), $545(\mathrm{E})$.

[14] A. González-Arroyo, C. López \& F.J. Ynduráin, Nucl. Phys. B153 (1979), 161.

[15] G. Curci, W. Furmanski \& R. Petronzio, Nucl. Phys. B175 (1980), 27.

[16] E.G. Floratos, D.A. Ross \& C.T. Sachrajda, Nucl. Phys. B152 (1979), 493.

[17] A. González-Arroyo \& C. López, Nucl. Phys. B166 (1980), 429.

[18] C. López \& F.J. Ynduráin, Nucl. Phys. B183 (1981), 157.

[19] W. Furmanski \& R. Petronzio, Phys. Lett. 97B (1980), 437.

[20] E. Remiddi \& J.A.M. Vermaseren, Int. J. Mod. Phys. A15 (2000), 725.

[21] J.A.M. Vermaseren, math-ph/0010025.

[22] J.A. Gracey, Phys. Lett. B488 (2000), 175.

[23] D.J. Broadhurst \& A.G. Grozin, Phys. Rev. D52 (1995), 4082.

[24] D.J. Broadhurst, Phys. Lett. B466 (1999), 319.

[25] G. Martinelli, C. Pittori, C.T. Sachrajda, M. Testa \& A. Vladikas, Nucl. Phys. B445 (1995), 81.

[26] E. Franco \& V. Lubicz, Nucl. Phys. B531 (1998), 641

[27] K.G. Chetyrkin \& A. Rétey, Nucl. Phys. B583 (2000), 3.

[28] M. Göckeler, R. Horsley, D. Pleiter, P.E.L. Rakow, A. Schäfer and G. Schierholz, hep-lat/0209160.

[29] M. Göckeler, R. Horsley, H. Oelrich, H. Perlt, D. Petters, P.E.L. Rakow, A. Schäfer, G. Schierholz \& A. Schiller, Nucl. Phys. B544 (1999), 699; S. Capitani, M. Göckeler, R. Horsley, H. Perlt, P.E.L. Rakow, G. Schierholz \& A. Schiller, Nucl. Phys. B593 (2001), 183.

[30] M. Guagnelli, K. Jansen, F. Palombi, R. Petronzio, A. Shindler \& L. Wetzorke, hep-lat/0303012.

[31] M. Lüscher, R. Narayanan, P. Weisz \& U. Wolff, Nucl. Phys. B384 (1992), 168.

[32] J.A. Gracey, hep-ph/0304113.

[33] S.G. Gorishny, S.A. Larin, L.R. Surguladze \& F.K. Tkachov, Comput. Phys. Commun. 55 (1989), 381.

[34] S.A. Larin, F.V. Tkachov \& J.A.M. Vermaseren, "The Form version of Mincer", NIKHEFH-91-18.

[35] P. Nogueira, J. Comput. Phys. 105 (1993), 279. 
[36] S.A. Larin \& J.A.M. Vermaseren, Phys. Lett. B303 (1993), 334.

[37] A. Hayashigaki, Y. Kanazawa \& Y. Koike, Phys. Rev. D56 (1997), 7350.

[38] F. Baldracchini, N.S. Craigie, V. Roberto \& M. Socolovsky, Fortschr. Phys. 30 (1981), 505.

[39] X. Artru \& M. Mekhfi, Z. Phys. C45 (1990), 669.

[40] W. Vogelsang, Phys. Rev. D57 (1998), 1886.

[41] J. Blümlein, Eur. Phys. J. C20 (2001), 683.

[42] J.C. Collins, Renormalization (Cambridge University Press, 1984).

[43] J.A. Gracey, Phys. Lett. B322 (1994), 141.

[44] A.N. Vasil'ev, Yu.M. Pis'mak \& J.R. Honkonen, Theor. Math. Phys. 46 (1981) 157; Theor. Math. Phys. 47 (1981) 291.

[45] A.N. Vasil'ev \& M.Yu. Nalimov, Theor. Math. Phys. 56 (1982), 643.

[46] T. van Ritbergen, J.A.M. Vermaseren \& S.A. Larin, Phys. Lett. B400 (1997), 379. 